\title{
La planificación estratégica y su incidencia en la competitividad de la microempresa del sector de muebles de madera del cantón Cuenca en la provincia del Azuay
}

\section{The strategic planning and its impact on the competitiveness of the micro firms of wood furniture sector in the canton Cuenca, province of Azuay}

Katina Vanessa Bermeo Pazmiño

Diego Marcelo Cordero Guzmán

Universidad Católica de Cuenca, Ecuador

Autor para correspondencia: kbermeo@ucacue.edu.ec, dcordero@ucacue.edu.ec

Fecha de recepción: 20 de julio de 2018 - Fecha de aceptación: 30 de agosto de 2018

Resumen: La investigación realiza el análisis de la influencia de la planificación estratégica sobre la competitividad de la microempresa del sector de fabricación de muebles de madera del cantón Cuenca en la provincia del Azuay en la República del Ecuador. Para ello, se elabora un modelo de análisis de competitividad, el mismo que está formado por tres constructos o variables. La primera variable relacionada con el proceso de planificación estratégica, con varios indicadores: el proceso de planeación en los dos últimos años, la estrategia básica, las políticas para la toma de decisiones, el trabajo en equipo para planificación, los objetivos específicos, el análisis FODA (fortalezas, oportunidades, debilidades, amenazas), la frecuencia de nuevos entrantes y el análisis de benchmarking. La segunda variable tiene que ver con la implementación de la estrategia, con sus indicadores: los cambios y logro de objetivos, asignación de recursos. Y una última variable que mide el nivel de competitividad alcanzado. La investigación arranca con el análisis de la literatura, apartado en donde se plantean las preguntas de investigación, objetivos e hipótesis; luego se propone el modelo cuya validación y comprobación se la ejecuta con ecuaciones estructurales; para al final elaborar los resultados, las conclusiones y sugerencias para futuras investigaciones.

Palabras Claves: planificación estratégica; competitividad; microempresa

Abstract: The research executes the analysis of the influence of strategic planning on the competitiveness of the micro firms of the wood furniture manufacturing sector of the canton Cuenca in the province of Azuay in the Republic of Ecuador. For this, a model of competitiveness analysis is elaborated and is formed by three constructs or variables. The first variable related to the strategic planning process, with some indicators: the planning process in the last two years, the basic strategy, the decision-making policies, the planning team work, the specific objectives, the SWOT analysis (strengths, opportunities, weaknesses, threats), frequency of new entrants, benchmarking analysis. The second variable about the implementation of the strategy, with its indicators: changes and achievement of objectives, allocation of resources. And one last variable that measures the level of competitiveness achieved. The research begins with the analysis of the literature, then the research questions, objectives and hypotheses are raised; then the model is proposed whose validation and verification is executed with structural equations, to elaborate the results, conclusions and recommendations for future researches.

Key Words: strategic planning; competitiveness; micro firms 


\section{Introducción}

Para Piñeiro (1993), la competitividad es un factor que pauta la diferencia en las organizaciones, éste no tiene límites precisos y fluctúa en función del entorno regional, el sector, la organización, el mercado, tipos de productos o servicios, en adición se debe destacar la capacidad de una nación para mantener su participación en mercados internacionales de modo que apoye a elevar el nivel de vida de los pobladores. En lo que va del Ecuador no se han determinado con exactitud los índices de competitividad de sus empresas, por ello que se ha vuelto preponderante ahondar en investigaciones serias sobre este tema, en razón de un 95\% de la dinámica comercial lo cubren la micro, pequeña y mediana empresa.

En el presente trabajo se toma como referencia inicial el Mapa de Competitividad del BID, conformado por ocho áreas claves dentro de la organización, las mismas que aportan a la generación de competitividad; entre éstas se encuentra la planificación estratégica, conformada por dos sub áreas: el proceso de planificación estratégica y la implementación de la estrategia; en adición se considera la variable a medir: la competitividad. Con estos tres componentes se propone el modelo empírico para análisis de competitividad para la microempresa del sector de fabricación de muebles de madera.

Con las tres variables descritas en el párrafo anterior se plantean dos hipótesis a ser probadas con la investigación, así: $\mathrm{H} 1$ : El proceso de planificación estratégica influye de manera positiva sobre la competitividad de la microempresa de fabricación de muebles de madera; $\mathrm{H} 2$ : La implementación de la estrategia influye de manera positiva sobre la competitividad de la microempresa de fabricación de muebles de madera. Con las dos hipótesis que constituyen una relación causal entre variables, se esquematiza el modelo de ecuaciones estructurales, de forma específica se usa la técnica PLS (Partial Least Square), o mínimos cuadrados parciales, que es de utilidad creciente en investigaciones de orden administrativo (Wold, 1979).

El modelo es testeado con datos levantados en 199 microempresas de fabricación de muebles de madera del cantón Cuenca de la provincia del Azuay, para ello se aplicó el instrumento tipo encuesta, tomado desde el Mapa de Competitividad desarrollado por el BID con preguntas o indicadores sobre planificación estratégica.

A partir de dicha prueba se presentan los resultados, en donde se valida que "el proceso de planificación estratégica" influencia de manera positiva sobre la competitividad, así como la" implementación de la estrategia". Se evidencia que el indicador que indaga si la empresa ha realizado un "proceso de planificación estratégica en los últimos dos años", no aporta a la variable que la incluye, esto implica que no es recomendable que forme parte del instrumento.

El análisis de los datos del modelo se ejecuta a través de estadísticos de ecuaciones estructurales con la herramienta para modelamiento SMART PLS 3.1.9. Al final se levantan las conclusiones y recomendaciones para futuras investigaciones. 


\section{Materiales y métodos}

\section{Marco teórico}

La competitividad identificada desde la teoría del comercio como motor del crecimiento de los países se respalda en los autores clásicos Adam Smith y David Ricardo, en donde identifican ventajas comparativas absolutas entre naciones y, en donde las fuerzas del mercado asignan recursos a los sectores mayormente productivos (Smith, 1776) y (Ricardo, 1817).

La evolución de la Teoría de la Competitividad inicia con la Teoría Económica Tradicional representada por el modelo de comercio internacional, en donde Smith pone especial atención a la nación; y la Teoría Económica Moderna identificada por el modelo de la ventaja competitiva de las naciones, también conocido como el "Diamante de la Ventaja Nacional", del que se derivan los determinantes de la ventaja nacional competitiva, y de cuyo proceso de evolución se obtienen los dos estudios más reconocidos en el ámbito mundial que miden la competitividad de las naciones mediante la ejecución de sus modelos teóricos, el Foro Económico Mundial y el Instituto Internacional para el Desarrollo de la Capacidad de Gestión (WEF e IMD por sus siglas en inglés) (Benzaquen, Del Carpio, Zegarra, \& Valdivia, 2010).

La competitividad es revisada desde dos enfoques: empresa o sector y entorno nacional, identificando los niveles micro y macro respectivamente; se determina que la competitividad es un fenómeno esencialmente del nivel micro influido por determinados parámetros del nivel macro (Ezeala-Harrison, 1999).

La competitividad se ha visto beneficiada de las nuevas tecnologías en la transferencia del conocimiento entre países y en el campo de la dirección estratégica global la importancia en los recursos intangibles y el conocimiento en la economía y en la sociedad son considerados procesos cada vez más significativos (Tuson, Ramos, \& Ramo, 1999).

Según (Scott \& Lodge, 1995) la competitividad es cada vez más un asunto de estrategias y estructuras y cada vez menos una consecuencia de las dotaciones naturales de un país. La competitividad debe ser visionaria y consecuencia de una planificación que permita el establecimiento de actividades y tareas con tiempos y responsables, para así ir creando una estructura eficiente de cumplimiento de estrategias planteadas.

El análisis moderno de la competitividad obliga a las organizaciones a operar dentro de comunidades más vastas, con las leyes y costumbres que ayudan a determinar la viabilidad de las nuevas direcciones estratégicas dentro de las empresas con impacto en la sociedad dentro de su actividad económica en una sociedad (Oster, 2000, págs. 4-128).

Las micro, pequeñas y medianas empresas se encuentran con la necesidad de dar respuestas estratégicas a los cambios y al nuevo ambiente de negocios globales. Estas respuestas deben tomar en cuenta cuatro aspectos claves: nuevas tendencias en los negocios, innovación, nuevas tecnologías de información y una gestión estratégica de la MIPYME (Lesakova, 2008). 
Michael Porter en su libro The Competitive Advantage of Nations de 1990 marca el estudio inicial de la competitividad desde los conceptos: Competencia, Estrategia Competitiva, Ventaja Competitiva, Competitividad, Clúster y Creación de Valor Compartido, y (Solvell, 2015) analiza tales conceptos partiendo de dicho estudio inicial y determinando las perspectivas actuales de la competitividad hacia la planificación estratégica dentro de las empresas.

La presencia de la MIPYME en América Latina es significativa, además que son consideradas como generadoras de empleo en la toda la región en un promedio del 64\%. Los recursos de las empresas son fuente para la construcción de ventajas competitivas, tomando en cuenta las estrategias globales de las empresas, los recursos humanos, tamaño, edad de la empresa y alianzas con el sector (Peña-Vinces, Acedo, \& Roldán, 2014).

El objetivo de analizar la relación entre la dimensión planificación estratégica y la competitividad en las MIPYMES como variables claves, es determinar el impacto que pueden tener en el desempeño empresarial. En el artículo de los autores (Mora-Riapira, Vera-Colina, \& Melgarejo-Molina, 2015) se evalúa el nivel de competitividad a partir del Mapa de Competitividad del Banco Interamericano de Desarrollo (BID) y a través del software SPSS, se infiere si la planificación estratégica tiene un impacto significativo en el funcionamiento de otras dimensiones de gestión organizacional de las MIPYME y su competitividad.

En el estudio Factores Internos que afectan a la competitividad en las empresas de la zona sur de Tamaulipas" de (Rodríguez, Bañuelos, Rubio, \& Chávez, 2010) se plantea el nivel de desempeño de los factores principales que inciden en la competitividad de las empresas: planificación estratégica, capacidades de marketing, calidad del producto o servicio, capacidades del máximo responsable o gerente y las tecnologías de la información y comunicación de la empresa.

La investigación Determinación de la Competitividad de la PYME en el nivel micro, el caso del Distrito Federal, México, establece el nivel competitivo de la micro, pequeña y mediana empresa evaluando aspectos internos de la misma como la planificación estratégica, producción y aprovisionamiento, calidad, comercialización, recursos humanos, contabilidad y finanzas, gestión ambiental y sistemas de información. Se utiliza la metodología del Mapa de Competitividad del BID y se concluye que las microempresas no son competitivas mientras que la pequeña y mediana son altamente competitivas, afirmando que la competitividad está relacionada con el tamaño y con el nivel de estudios del empresario (Saavedra García, Milla Toro, \& Tapia Sánchez, Determinación de la Competitividad de la PYME en el nivel micro. El caso del Distrito Federal, México, 2013).

El Mapa de Competitividad del BID identifica ocho áreas claves en la empresa y el micro y pequeño empresario se interesa de sobre manera en conocer qué áreas se relacionan y cuáles deberá atender simultáneamente dentro de su empresa. El empresario ubica erróneamente a la Planificación Estratégica como un área fuera del análisis global del resto de los factores y no la considera como un área independiente (Bermeo, 2016). La figura No. 1 ilustra la opinión de los encuestados sobre las áreas analizadas dentro de la micro y pequeña empresa. 


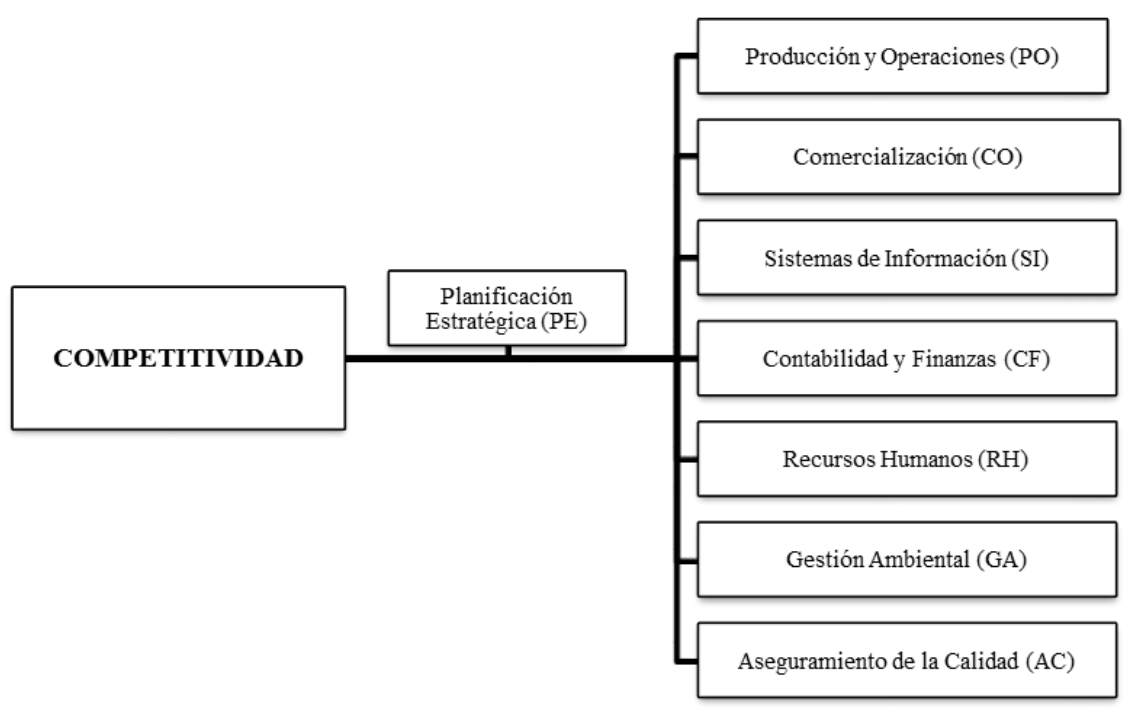

Figura No.1. Ilustración de opiniones de encuestados sobre áreas analizadas en la empresa FUENTE: (Bermeo, 2016).

El Censo Económico del Ecuador realizado el pasado año 2010 por el Instituto Ecuatoriano de Estadísticas y Censos -INEC-, muestra un total de 2.059.504.00 personas trabajando dentro de las actividades definidas por la Clasificación Industrial Internacional Uniforme-CIIU- ${ }^{1}$.

La investigación se aplica a la actividad fabricación de muebles de madera dentro de la industria manufacturera en la Provincia del Azuay, Cantón Cuenca. La Tabla No. 1 muestra el caso del sector manufacturero por tamaño de empresa,

Tabla No. 1. Industria Manufacturera por MIPYME

\begin{tabular}{lrr}
\hline Tamaño empresa & Industria manufacturera (\#) & Porcentaje de participación \\
\hline Micro empresa & 49.252 & $87.85 \%$ \\
Pequeña empresa & 5.209 & $9.29 \%$ \\
Mediana empresa & 1.033 & $1.84 \%$ \\
Grande empresa & 572 & $1.02 \%$ \\
TOTAL & $\mathbf{5 6 . 0 6 6}$ & $\mathbf{1 0 0 . 0 0 \%}$ \\
\hline
\end{tabular}

Fuente: Instituto Nacional de Estadísticas y Censos

Elaboración: Propia

Según información del mismo Censo, el Ecuador cuenta con numerosas y crecientes especies maderables gracias a su ubicación geográfica, esto hace que la materia prima que se requiera sea totalmente local y los muebles que el país produce sean elaborados con los materiales más diversos a fin de satisfacer las demandas variadas de los mercados nacionales e internacionales. El mercado de muebles de madera es muy importante para la economía del país; a la ciudad de Cuenca le corresponde el $60 \%$ de la producción total convirtiéndose en un sector valioso y vital para la ciudad.

\footnotetext{
${ }^{1}$ CIIU 4. Siglas del Código de Clasificación Industrial Internacional Uniforme de todas las actividades económicas. Cuarta Revisión. Elaborado por la Organización de las Naciones Unidas.
} 
Sobre el instrumento de medición utilizado en este artículo, es adaptado, diseñado y validado por (Saavedra García, Hacia la Determinación de la Competitividad de la PYME Latinoamericana, 2014) basado en el Mapa de Competitividad del Banco Interamericano de Desarrollo, el cual se define como una herramienta de diagnóstico que se aplica en la empresa y permite conocer su estado inicial frente a la competitividad e identificar las áreas en las que presenta fortaleza y aquellas en las que tiene debilidad, con el propósito final de desarrollar planes de acción, que conduzcan al mejoramiento de su competitividad. Dicho instrumento de medición identifica ocho áreas claves de la empresa: Planificación Estratégica, Producción y Operaciones, Aseguramiento de la Calidad, Comercialización, Contabilidad y Finanzas, Recursos Humanos, Gestión Ambiental y Sistemas de Información (Bernal Rodríguez, 2013). La Planificación Estratégica es el área validada frente a la Competitividad y cuyos resultados se muestran en este artículo.

\section{Metodología}

\section{Planteamiento del problema de investigación}

El problema de investigación es concebir el Modelo para análisis de competitividad, el mismo que está formado por tres constructos o variables: el proceso de planificación estratégica, la implementación de la estrategia, la competitividad. 


\section{Formulación del problema}

Se adjunta la Tabla No.2, donde consta la llamada matriz de congruencia con las preguntas de investigación (planteadas desde el análisis de la literatura) conjuntamente con los objetivos e hipótesis, en una relación uno a uno.

Tabla No. 2. Matriz de congruencia, preguntas, objetivos e hipótesis

\begin{tabular}{|c|c|c|}
\hline Preguntas de Investigación & Objetivos & Hipótesis \\
\hline $\begin{array}{l}\text { ¿Cómo influye el proceso de } \\
\text { planificación estratégica sobre la } \\
\text { competitividad de la } \\
\text { microempresa de fabricación de } \\
\text { muebles de madera? }\end{array}$ & $\begin{array}{l}\text { Determinar la influencia del proceso } \\
\text { de planificación estratégica sobre la } \\
\text { competitividad de la microempresa } \\
\text { de fabricación de muebles de } \\
\text { madera. }\end{array}$ & $\begin{array}{l}\text { Hipótesis } 1 \text { (H1): } \\
\text { El proceso de planificación } \\
\text { estratégica influye de manera directa } \\
\text { sobre la competitividad de la } \\
\text { microempresa de fabricación de } \\
\text { muebles de madera. }\end{array}$ \\
\hline $\begin{array}{l}\text { ¿Cómo influye la implementación } \\
\text { de la estrategia sobre la } \\
\text { competitividad de la } \\
\text { microempresa de fabricación de } \\
\text { muebles de madera? }\end{array}$ & $\begin{array}{l}\text { Determinar la influencia de la } \\
\text { implementación de la estrategia } \\
\text { sobre la competitividad de la } \\
\text { microempresa de fabricación de } \\
\text { muebles de madera. }\end{array}$ & $\begin{array}{l}\text { Hipótesis } 2 \text { (H2): } \\
\text { La implementación de la estrategia } \\
\text { influye de manera directa sobre la } \\
\text { competitividad de la microempresa } \\
\text { de fabricación de muebles de } \\
\text { madera. }\end{array}$ \\
\hline
\end{tabular}

\section{Modelo propuesto}

El Modelo de la investigación es de ecuaciones estructurales (MEE), que Para Fornell y Larcker (1981), tienen relevancia para análisis multivalente y que según Céspedes Lorente (1996), son muy factibles de aplicarse en las ciencias sociales, sobre todo en las áreas de la administración de empresas. En la investigación se hace uso de la técnica PLS (Partial Least Square) o mínimos cuadrados parciales, que es un tipo de MEE y cuya validación se la ejecuta con dos componentes según Cepeda y Roldán (2004):

- El modelo de medida, en donde se determinan las cargas factoriales de las variables observables (indicadores) en relación a sus variables latentes (constructos).

- El modelo estructural, en donde se validan las relaciones de tipo causal entre los constructos y que están definidas como hipótesis.

A partir de las dos hipótesis planteadas, se propone el modelo indicado o en la Figura No.2, en donde constan tres constructos:

1. Proceso: hace referencia al proceso de planificación estratégica

2. Inplementac.: es la implementación de la estrategia

3. Competitividad: el índice de competitividad de la microempresa de fabricación de muebles de madera. 


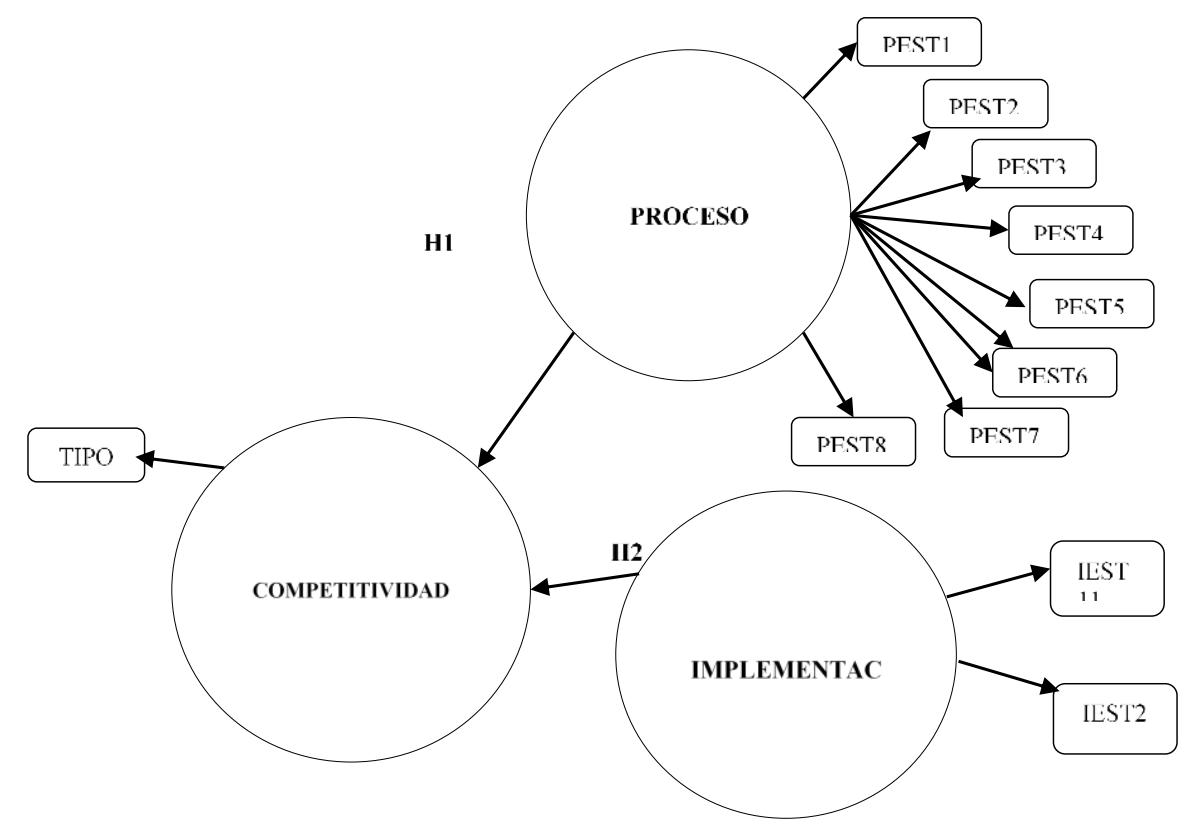

Figura No.2. Modelo para análisis de Competitividad en función de la Planificación Estratégica

\section{Población y muestra}

Para determinar la muestra, se toma como referencia las características del modelo propuesto (11 indicadores, 3 constructos de los cuales 2 de ellos apuntan a una variable dependiente). De acuerdo a la "modelización PLS", el tamaño muestral para el modelo es de $10 * 2=20$, (Cordero, 2016). Es decir que son suficientes 20 encuestas para probar el modelo, a pesar de ello se aplicaron 199, para dar mayor consistencia a los resultados.

\section{Instrumento para Recolección de Información}

Se toma el instrumento con 11 preguntas (indicadores), sobre los constructos o variables que pertenecen a planificación estratégica, como se indica en la Tabla No.3. Es evidente que el instrumento es el resultado de la operacionalización de las variables y es parte del Mapa de Competitividad del BID, y se evalúa a través de una escala de likert de 0 a 5.

Tabla No 3. Instrumento para captura de datos

\begin{tabular}{|c|c|c|}
\hline ID & Ítems & Escala \\
\hline 1. & PROCESO DE PLANIFICACIÓN ESTRATÉGICA & \\
\hline \multirow[t]{2}{*}{ PEST1 } & ¿La empresa ha realizado un proceso de planeación estratégica en los últimos dos años? & \\
\hline & & $\begin{array}{llll}0 & 1 & 2 & 3 \\
4 & 5 & & \end{array}$ \\
\hline PEST2 & $\begin{array}{l}\text { ¿La empresa tiene una estrategia básica de negocios escrita y conocida por todos los que deben } \\
\text { ejecutarla? }\end{array}$ & $\begin{array}{llll}0 & 1 & 2 & 3 \\
4 & 5 & & \end{array}$ \\
\hline PEST3 & $\begin{array}{l}\text { ¿La empresa tiene como política para la toma de decisiones involucrar a las personas } \\
\text { responsables en su ejecución y cumplimiento? }\end{array}$ & $\begin{array}{llll}0 & 1 & 2 & 3 \\
4 & 5 & & \end{array}$ \\
\hline
\end{tabular}


PEST4 ¿La planeación estratégica es el resultado de un trabajo en equipo y participan en su elaboración quienes son responsables por su ejecución y cumplimiento?

PEST5 ¿Se definen objetivos específicos, cuantificables y medibles, junto con un plazo de tiempo definido para su ejecución, por parte de las personas responsables del área o departamento involucrados?

PEST6 ¿Al planear se desarrolla un análisis FODA (Fortalezas, Oportunidades, Debilidades y 001123 Amenazas) para la empresa y el sector donde ésta ópera, con la adecuada participación de las 45 áreas?

PEST7 ¿Se analiza con frecuencia el sector donde opera la empresa considerando entre otros factores: 012123 nuevos proveedores, nuevos clientes, nuevos competidores, nuevos productos competidores, 45 nuevas tecnologías y nuevas regulaciones?

PEST8 ¿Al formular las estrategias competitivas, se utiliza la técnica de comparar la empresa con las $0 \begin{array}{lll}0 & 2 & 3\end{array}$ mejores prácticas ("benchmarking")?

2. IMPLEMENTACIÓN DE LA ESTRATEGIA

IEST1 ¿El personal está activamente involucrado en el logro de los objetivos de la empresa, así como $0 \begin{array}{lllll}2 & 2\end{array}$ en los cambios que demanda la implementación de la estrategia? $\quad 45$

IEST2 ¿La planeación estratégica da las pautas para la asignación general de recursos en cada área 01223 del negocio de la empresa, con un seguimiento efectivo?

3. COMPETITIVIDAD

TIPO ¿El nivel de competitividad actual es ? 123

\section{Resultados y discusiones}

\section{Proceso de la información}

- Se toma la información del Censo sobre el sector maderero a la actividad fabricación de muebles de madera en la Provincia del Azuay, Cantón Cuenca, como fuente de aplicación de las encuestas.

- A través de la participación de encuestadores se colectan los datos.

- Los datos de las encuestas luego de su depuración son cargados en el software de procesamiento estadístico SPSS.

- El modelo estructural se diseña e implementa con el software Smart PLS 3.1.9

- Los datos en SPSS, son exportados a Smart PLS 3.1.9.

- Con el modelo generado en Smart PLS 3.1.9, se elaboran los reportes de los estadísticos, como se indica en la figura No.2.

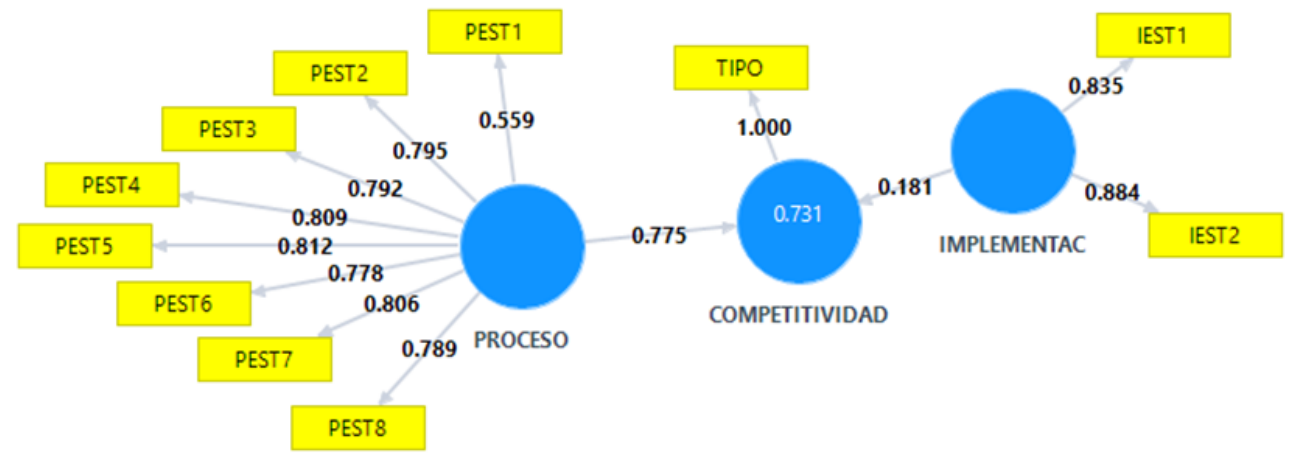

Figura No. 3. Resultados del Modelo Estructural 
El modelo es analizado a través de dos fases:

Validez y Fiabilidad del Modelo de Medida

Aquí se valida si los conceptos teóricos son soportados a través de las variables observadas; en la Figura No. 2 se ilustran los resultados del modelo estructural, en donde las variables observadas o indicadores son: PEST1, PEST2, PEST3, PEST4, PEST5, PEST6, PEST7, PEST8, IEST1, IEST2 y TIPO; son variables latentes o constructos: proceso, implementac, competitividad. En la Tabla No.4 se desglosan los valores de esta evaluación.

Tabla No 4. Fiabilidad del modelo de medida

\begin{tabular}{|c|c|}
\hline Parámetros & Valores Obtenidos \\
\hline $\begin{array}{l}\text { Fiabilidad } \\
\text { individual del } \\
\text { ítem. }\end{array}$ & $\begin{array}{l}\text { Todas las cargas están por encima de } 0.7 \text {, a excepción del indicador PEST1 como se indica } \\
\text { en la Figura No.2. Lo que implica que este indicador no aporta al constructo. }\end{array}$ \\
\hline $\begin{array}{l}\text { Fiabilidad de } \\
\text { cada constructo }\end{array}$ & $\begin{array}{l}\text { Para que cada constructo sea considerado como fiable, su alfa Cronbach debe superar el } \\
\text { valor 0.7. Para el modelo todos los constructos resultan fiables como se indica en la Tabla } \\
\text { No.5. } \\
\text { En el análisis de fiabilidad compuesta, todos los constructos del modelo presentan valores } \\
\text { superiores a } 0.6 \text {, confirmándose por tanto la consistencia interna de todos los constructos, } \\
\text { como se indica en la Tabla No.6. }\end{array}$ \\
\hline $\begin{array}{l}\text { Validez } \\
\text { convergente }\end{array}$ & $\begin{array}{l}\text { Para evaluar la validez convergente de los constructos se hace uso de la varianza extraída } \\
\text { media (AVE). Los valores para el indicador AVE, superan el valor mínimo recomendable de } \\
0.5 \text {, como se indica en la Tabla No.7. }\end{array}$ \\
\hline $\begin{array}{l}\text { Validez } \\
\text { discriminante }\end{array}$ & $\begin{array}{l}\text { Con el chequeo de cargas cruzadas, se valida que cada indicador tiene correlación con su } \\
\text { propia variable latente antes que, con otras variables, como se indica en la Tabla No.8.Pues } \\
\text { el valor de cada celda marcada es mayor que el resto de elementos de la fila. Así, para el } \\
\text { caso de IEST1 vs IMPLEMENTAC, resulta que } 0.928 \text { es mayor que } 0.379 \text { y } 0.272 \text {. }\end{array}$ \\
\hline
\end{tabular}

Tabla No 5. Fiabilidad del constructo

\begin{tabular}{ll}
\hline & Cronbachs Alpha \\
\hline COMPETITIVIDAD & 1.000 \\
IMPLEMENTAC & 0.850 \\
PROCESO & 0.919
\end{tabular}

Tabla No 6. Fiabilidad compuesta

\begin{tabular}{lr}
\hline & Composite Reliability \\
\hline COMPETITIVIDAD & 1.000 \\
IMPLEMENTAC & 0.930 \\
PROCESO & 0.935 \\
\hline
\end{tabular}

Tabla No. 7. Validez convergente

\begin{tabular}{lr}
\hline & AVE \\
\hline & AVE \\
COMPETITIVIDAD & 1.000 \\
IMPLEMENTAC & 0.869
\end{tabular}




\begin{tabular}{ll}
\hline PROCESO & 0.644
\end{tabular}

Tabla No 8. Cargas cruzadas. Validez discriminante

\begin{tabular}{lrrr}
\hline & COMPETITIVIDAD & IMPLEMENTAC & PROCESO \\
\hline IEST1 & 0.379 & 0.928 & 0.272 \\
IEST2 & 0.401 & 0.936 & 0.309 \\
PEST1 & 0.469 & 0.070 & 0.571 \\
PEST2 & 0.666 & 0.172 & 0.782 \\
PEST3 & 0.664 & 0.337 & 0.790 \\
PEST4 & 0.678 & 0.387 & 0.838 \\
PEST5 & 0.681 & 0.342 & 0.872 \\
PEST6 & 0.652 & 0.215 & 0.826 \\
PEST7 & 0.676 & 0.186 & 0.857 \\
PEST8 & 0.662 & 0.249 & 0.844 \\
TIPO & 1.000 & 0.418 & 0.806 \\
\hline
\end{tabular}

Valoración del modelo estructural, se lo ejecuta en base a la evaluación del peso y la magnitud de las relaciones entre los distintos constructos. En la Tabla No.9, se desglosan los datos de esta evaluación, así: el índice R2, el efecto f 2, los coeficientes path estandarizados $\beta$ y el análisis de Bootstrapping.

Tabla No.9. Evaluación del modelo estructural

\begin{tabular}{ll}
\hline \multicolumn{1}{c}{ Parámetro } & \multicolumn{1}{c}{ Valores obtenidos del modelo } \\
\hline Índice $\boldsymbol{R}^{\mathbf{2}}$ & El poder predictivo del modelo para las variables latentes dependientes, en este caso \\
& COMPETITIVIDAD, se obtiene con el índice R ${ }^{2}$, cuyo valor es mayor a 0.1, lo que ratifica \\
& la característica predictiva del modelo. Como se indica en la Tabla No.10. \\
Efecto $\boldsymbol{f}^{\mathbf{2}}$ & $\begin{array}{l}\text { Mide el impacto sobre un constructo dependiente de una variable latente, para el modelo } \\
\text { se presentan valores que se encuentran en el rango permisible. Como se indica en la Tabla }\end{array}$ \\
& No.11. \\
Coeficientes path & Para el modelo, un coeficiente no supera el valor mínimo de 0.2, razón por la cual desde el \\
estandarizados $\boldsymbol{\beta}$ & punto de vista estructural el modelo debería ser reorganizado. Tabla No.12. \\
Análisis de & Bootstrap también determina el cálculo del error estándar de los parámetros y los valores \\
Bootstrapping & "t" de Student; en este ámbito se consideran como significativos a los indicadores cuyo "t" \\
& de Student es mayor que 1,96. Todos los valores son superiores a 1,96, como se indica en \\
\hline
\end{tabular}

Tabla No.10. R2 de las variables latentes dependientes

\begin{tabular}{rr}
\hline & R Square \\
\hline COMPETITIVIDAD & 0.681 \\
\hline
\end{tabular}

Tabla No. 11. F 2 de las variables latentes dependientes

\begin{tabular}{|c|c|c|c|c|c|}
\hline \multirow{2}{*}{ COMPETITIVIDAD } & COMPETITIVIDAD & ENTRADA & PLANSIST & PROCESOS & SALIDAS \\
\hline & & & & & \\
\hline ENTRADA & 0.000 & & & & \\
\hline PLANSIST & 0.005 & & & & \\
\hline
\end{tabular}




\begin{tabular}{ll}
\hline PROCESOS & 0.013 \\
SALIDAS & 0.049
\end{tabular}

La Tabla No.12 despliega las relaciones entre los constructos del modelo a través de los caminos beta estandarizados, el error estándar, el valor de t de student, el nivel de significancia y la aceptación o rechazo de la hipótesis.

Tabla No 12. Relaciones entre constructos

\begin{tabular}{lrlccccc}
\hline & $\boldsymbol{\beta}$ & $\begin{array}{l}\text { Error } \\
\text { estándar }\end{array}$ & $\begin{array}{c}\mathbf{t}- \\
\text { student }\end{array}$ & & $\begin{array}{c}\text { Nivel de } \\
\text { Valores } \\
\text { significanc } \\
\text { ia }\end{array}$ & $\begin{array}{c}\text { Aceptaci } \\
\text { ón o No } \\
\text { Aceptaci } \\
\text { ón }\end{array}$ \\
\hline IMPLEMENTAC -> & & & & & & & \\
COMPETITIVIDAD & 0.18 & 0.037 & 4.947 & 0.000 & $* * *$ & Se acepta \\
PROCESO -> COMPETITIVIDAD & $\begin{array}{r}0.74 \\
9\end{array}$ & 0.028 & 26.699 & 0.000 & & \\
& 9 & & & & $* * *$ & Se acepta \\
\hline
\end{tabular}




\section{Conclusiones y discusión}

De acuerdo a la matriz de congruencia planteada en donde se identificaron las preguntas, los objetivos y las hipótesis se concluye que:

- El proceso de planificación estratégica influye de manera positiva sobre la competitividad de la microempresa de fabricación de muebles de madera.

- La implementación de la estrategia influye de manera positiva sobre la competitividad de la microempresa de fabricación de muebles de madera.

De acuerdo a los resultados del "modelo de medida", se comprueba que se cumplen con la condición de validación. Esto implica que el modelo es válido y confiable, por tanto, la teoría está completamente sustentada y el instrumento es totalmente válido, salvo por una pregunta que debería ser reformulada o eliminada que corresponde al indicador PEST1. Pues en efecto la planificación estratégica tiene un horizonte de ejecución de cinco años más no de dos años.

El modelo estructural es válido, la magnitud de las relaciones entre los distintos constructos da certeza de que las hipótesis son verdaderas, en adición que el modelo es altamente predictivo.

A pesar de que en primera instancia se podría suponer que la microempresa no ejecuta planificación estratégica, resulta evidente que ésta es crítica en importancia para generar competitividad.

\section{Agradecimientos}

A la Universidad Católica de Cuenca por la apertura a procesos investigativos. 


\section{Bibliografía}

Benzaquen, J., Del Carpio, L., Zegarra, L., \& Valdivia, C. (2010). Un Índice Regional de Competitividad para un País. Revista CEPAL 102, 69-86.

Bermeo, V. (2016). Determinación de la Competitividad Sistémica de la MIPYME Manufacturera en Ecuador: El caso de la fabricación de muebles de madera en el área urbana del cantón Cuenca de la Provincia del Azuay, Ecuador. Tesis de Grado Doctoral. México.

Bernal Rodríguez, E. (2013). Hacia la Competitividad Sistémica de las MiPYMES. Un análisis del contexto colombiano. . Revista Ensayos, 43-59.

Cepeda, G., \& Roldán, J. L. (2004). Aplicando en la práctica la técnica PLS en la Administración de Empresas. Sevilla: Facultad de Ciencias Económicas y Empresariales.

Céspedes Lorente, J. J., \& Sánchez Pérez, M. (1996). Tendencias y desarrollos recientes en métodos de investigación y análisis de datos en dirección de empresas. Revista Europea de Dirección y Economía de la Empresa, 5(3), 23-40.

Cordero, D. (2016). Modelo para Gobierno de Tecnologías de la Información (GTI): caso de las Universidades Cofinanciadas de la Zona 6 de la República del Ecuador (Tesis de Grado Doctoral). México.

Ezeala-Harrison, F. (1999). Theory and Policy of International Competitiveness. Londres: Cengage Learning.

Fornell, C., \& Larcker, D. F. (February de 1981). Evaluating Structural Equation Models with Unobservable Variables and Measurement Error. Journal of Marketing Research, 18(1), 39-50.

Lesakova, L. (2008). Influence of globalization on small and medium enterprises. Ekonomicky Casopis.

Mora-Riapira, E., Vera-Colina, M., \& Melgarejo-Molina, Z. (2015). Planificación Estratégica y Niveles de Competitividad de las MiPYMES del sector comercio en Bogotá. Estudios Gerenciales, 31, 79-87.

Oster, S. (2000). Análisis Moderno de la Competitividad. Oxford University Press.

Peña-Vinces, J., Acedo, F., \& Roldán, J. (2014). Model of the International Competitiveness of SMNEs for Latin American Developing Countries. European Business Review, 26, 552 567.

Piñeiro, M. (1993). Innovation, Competitiveness and Agroindustrial Development. Economic Development. 
Ricardo, D. (1817). On the Principles of Political Economy and Taxation. Cambridge Universtity.

Rodríguez, A., Bañuelos, F., Rubio, B., \& Chávez, J. (2010). Factores Internos que afectan a la Competitividad en las empresas de la zona sur de Tamaulipas. Revista de la Ingeniería Industrial.

Saavedra García, M. (2014). Hacia la Determinación de la Competitividad de la PYME Latinoamericana. Ciudad de México: Publicaciones Empresariales UNAM. FCA Publishing. .

Saavedra García, M., Milla Toro, S., \& Tapia Sánchez, B. (2013). Determinación de la Competitividad de la PYME en el nivel micro. El caso del Distrito Federal, México. . FAEDPYME International Review, 2, 18-32.

Scott, B., \& Lodge, G. (1995). US Competitiveness in the World Economy.

Smith, A. (1776). The Wealth of Nations. Escocia.

Solvell, O. (2015). The Competitive Advantage of Nations 25 years - Opening up new perspectives on Competitiveness. . Competitiveness Review, 25, 471-481.

Tuson, A., Ramos, S., \& Ramos, R. (1999). Nuevas tendencias tecnológicas de sistemas de información inteligentes orientadas al sector empresarial.

Wold, H. (1979). Model Construction and Evaluation when Theoretical Knowledge Is Scarce: An Example of the Use of Partial Least Squares. University de Genève. Genève: Cahiers du Department D’Économétrie. 